Revue internationale P.M.E.

Économie et gestion de la petite et moyenne entreprise

\title{
Les comportements d'intégration marketing stratégie et leur influence sur la performance de la petite entreprise: un cadre conceptuel préliminaire
}

\section{Bertrand Saporta et Catherine Lapassouse Madrid}

Volume 8, numéro 2, 1995

URI : https://id.erudit.org/iderudit/1008353ar

DOI : https://doi.org/10.7202/1008353ar

Aller au sommaire du numéro

Éditeur(s)

Presses de l’Université du Québec

ISSN

0776-5436 (imprimé)

1918-9699 (numérique)

Découvrir la revue

Citer cette note

Saporta, B. \& Lapassouse Madrid, C. (1995). Les comportements d'intégration marketing stratégie et leur influence sur la performance de la petite entreprise: un cadre conceptuel préliminaire. Revue internationale P.M.E., 8(2), 147-174. https://doi.org/10.7202/1008353ar
Résumé de l'article

Cet article présente une réflexion sur le thème de la cohérence entre les décisions stratégiques et marketing dans les PME. Les auteurs abordent tout d'abord le sujet d'une manière conceptuelle en procédant à une revue des différentes contributions de la littérature en stratégie et en marketing. Le constat d'une connaissance insuffisante de ces mécanismes d'intégration les conduit ensuite à proposer un cadre méthodologique destiné à observer les comportements d'intégration stratégie/marketing dans le processus de décision entrepreneurial. 


\title{
Les comportements d'intégration marketing stratégie et leur influence sur la performance de la petite entreprise: un cadre conceptuel préliminaire
}

\author{
Bertrand SAPORTA* \\ Catherine LAPASSOUSE MADRID** \\ Université Bordeaux I
}

MOTS CLÉS
Marketing - Stratégie - Cohérence - Intégration fonctions
Recherche - Méthodologie - Projet

\begin{abstract}
RÉSUMÉ
Cet article présente une réflexion sur le thème de la cohérence entre les décisions stratégiques et marketing dans les PME. Les auteurs abordent tout d'abord le sujet d'une manière conceptuelle en procédant à une revue des différentes contributions de la littérature en stratégie et en marketing. Le constat d'une connaissance insuffisante de ces mécanismes d'intégration les conduit ensuite à proposer un cadre méthodologique destiné à observer les comportements d'intégration stratégie/marketing dans le processus de décision entrepreneurial.
\end{abstract}

* Bertrand Saporta, professeur de sciences de gestion à l'Université de Bordeaux I, est directeur du CREGE, laboratoire de recherche en gestion de cette université et coresponsable du DESS «Création d'entreprises et gestion de projets innovants ». Outre de nombreux articles, il a publié deux ouvrages : Stratégies pour la PME, Éd. Montchrestien, 1986 et Marketing industriel, Éd. Eyrolles, 1989. Ses domaines de recherche privilégiés sont la stratégie d'entreprise appliquée aux PME, le marketing industriel et l'entrepreneuriat. Il exerce également des activités de consultation auprès de PME d'Aquitaine spécialisées dans les biens industriels. Adresse : Centre de recherches et d'études en gestion, Faculté des sciences économiques et de gestion, Université Bordeaux I, avenue Léon-Duguit, 33604 Pessac, Cedex, France.

** Catherine Lapassouse Madrid est maître de conférences en sciences de gestion à l'Université de Bordeaux I, IUT, Département techniques de commercialisation. Ses préoccupations de recherche portent plus particulièrement sur les spécificités du marketing appliqué aux PME, notamment dans le cadre de leur développement international. Elle a publié des articles sur ce thème ainsi que sur celui de l'évolution des circuits de distribution des produits industriels. Adresse: Département techniques de commercialisation, Université Bordeaux I, avenue Léon-Duguit, 33604 Pessac, Cedex, France. 


\begin{abstract}
Integration of strategic and marketing decisions in SMBs: propositions for a conceptual frame and empirical verification processes. This article presents remarks about the coherence between strategic and marketing decisions in SMBs. First, the authors review the contributions of strategic and marketing literature and emphasize the inadequate knowledge of integration mechanisms. Therefore, they propose a methodological approach in order to observe such behaviours among decisions processes in SMBs.

\section{RESUMEN}

Integracion de las decisiones de estrategica y de marketing en el proceso de decision empresarial: proposicion por un conceptual marco y una empirica verificacion. Esta articulo presenta una reflexion en cuento a la coherencia entre las decisones de estrategica y de marketing en la PyMEs. Primero, los autores abordan el tema de maniera conceptual al procedan al examen de las diferentes contribuciones de la literatura de estrategica y de marketing. La conprobacion de conocimiento insuficiente de estos mecanismos de intagracion los lleva despues a proponer un marco metodologico destinado a observar los comportamentos de integracion en el proceso de decision empresarial.
\end{abstract}

\title{
Introduction
}

La nécessité d'une cohérence entre les décisions stratégiques et les décisions marketing est un thème rarement traité de manière explicite, dans la mesure où ces deux catégories de décisions sont souvent confondues, ou du moins difficiles à distinguer, par ces dirigeants. Ce risque est particulièrement élevé lorsqu'on met en parallèle, d'une part, les décisions stratégiques proprement dites de l'entreprise (diversification, exportation, intégration verticale) et, d'autre part, les décisions marketing de caractère stratégique ou stratégies marketing, qui en découlent (choix de canaux de distribution, de segmentation ou de positionnement), par opposition à des décisions de caractère plus opérationel ou quotidien (recrutement de vendeurs, modifications mineures du programme marketing). On a alors affaire à des domaines de décision voisins, mais distincts, comme on le verra par la suite.

Une réflexion approfondie sur cette question apparaît d'autant plus nécessaire qu'on peut supposer que la performance des petites et moyennes entreprises dépend de l'habileté avec laquelle leurs responsables évitent de tomber dans ce type de confusion, tout en assurant un minimum de cohérence entre les deux catégories de décisions. Peu de chercheurs en donnent une vérification empirique; on peut citer toutefois un certain nombre d'études qui semblent lier la performance des PME planificatrices non pas tant au degré de formalisation de leur processus de planification stratégique qu'au soin avec lequel leurs dirigeants font converger le plan stratégique et les différentes 
fonctions de l'entreprise (Bracker et Pearson, 1986; Bracker, Keats et Pearson, 1988 ; Robinson, Pearce et al., 1984).

Nous aborderons tout d'abord le sujet d'un point de vue documentaire et conceptuel. Après avoir examiné comment le thème de la cohérence entre stratégie et marketing est abordé dans la littérature générale de gestion (section 1), on cherchera à répondre à la question de savoir si cette cohérence ne va pas davantage de soi pour un dirigeant de petite entreprise qu'au sein d'une grande organisation (section 2). Nous serons ensuite amenés à proposer un cadre méthodologique destiné à faire avancer la recherche dans ce domaine (section 3 ).

\section{Une approche conceptuelle et documentaire des liens entre stratégie et marketing}

On examinera, dans un premier temps, comment le problème est traité en dehors de tout contexte entrepreneurial ou spécifique à la petite entreprise. Ces premiers éléments d'information seront complétés par une analyse du même problème lorsqu'une seule et même personne - l'entrepreneur ou dirigeant-propriétaire - est le siège de cette recherche indispensable de cohérence.

Alors que la nature des liens qu'entretient la stratégie avec la finance est périodiquement examinée par les auteurs (pour un exemple récent, voir Hirigoyen, 1993), il n'en est pas de même de ses rapports avec le marketing, qui sont souvent traités de manière allusive. On peut malgré tout isoler quelques points de repère utiles dans les deux disciplines concernées.

\subsection{Les angles d'approche de la littérature stratégique}

Quel que soit le mot clé utilisé, la littérature stratégique nous fournit moins un traitement précis du problème abordé que des éléments de réflexion pour nos développements ultérieurs, notamment pour la construction d'un modèle de recherche empirique.

Le concept générique de stratégie a fait ces dernières années l'objet de remises en cause globales, utilisant notamment une perspective historique (Martinet, 1988; Mintzberg, 1990 ; Porter, 1991). Le fil conducteur de ces travaux est que l'approche «classique» de la stratégie - celle des «pères fondateurs » de l'École de Harvard - sous-entendait un jeu d'hypothèses restrictives qui ne sont pas forcément remplies dans les environnements et les organisations d'aujourd'hui. Dans cette conception, l'intégration à la stratégie des principales fonctions ne posait pas de problème. Elle constituait même l'une de ses principales justifications, la stratégie étant censée intégrer toutes les fonctions de l'entreprise, y compris le marketing, en lui fournissant un 
ensemble d'objectifs et de politiques destinés à les empêcher d'œuvrer dans des directions centrifuges (Porter, op. cit., p. 96). Mais cette conception de la stratégie, apparentée à la formulation d'un "grand dessein » consciemment concocté par le stratège, est-elle tenable aujourd'hui, lorsque l'on énumère les postulats qui l'accompagnent, notamment la séparation entre la phase de formulation de la stratégie et celle de sa mise à exécution, ainsi que la possibilité d'un contrôle étroit de la deuxième phase par la personne en charge de la première ? Ce dernier postulat n'est-il pas plutôt représentatif d'un style de direction «entrepreneurial», où tous les pouvoirs sont concentrés en une même personne, mais dont le caractère fondamentalement intuitif échappe par nature au cadre stratégique classique (Mintzberg, 1990, p. 186)?

La planification stratégique peut être définie comme une procédure formalisée de prise de décision par laquelle une entreprise fixe les grandes orientations de son développement (Stratégor, 1988). Même si cette conception assez classique de la stratégie est actuellement très controversée, elle fournit cependant un cadre de réflexion assez proche de nos préoccupations dans la mesure où elle traite explicitement de la relation entre stratégie et fonctions de l'entreprise, ne serait-ce qu'au travers de la distinction entre planification stratégique et planification opérationnelle. Si l'on isole le marketing des autres fonctions, on peut montrer ses relations avec la stratégie, en décomposant les principales étapes de la planification stratégique, et en soulignant les apports du marketing à chacune de ces étapes (voir figure 1). L'apport le plus fondamental se situe au niveau de l'exécution du plan stratégique (phase terminale du schéma): le succès d'une option stratégique dépend en grande partie de l'habileté avec laquelle les commerciaux cultiveront les marchés cibles privilégiés par l'option. Mais on voit qu'au cours des étapes antérieures, la contribution du marketing reste significative (la liste des apports indiqués dans le schéma n'est pas limitative).

Le management stratégique, une approche «rénovée» de la stratégie, cherche à en combler ses lacunes, en privilégiant la dimension organisationnelle des choix stratégiques, en considérant les stratégies «émergentes » sur le même plan que les choix stratégiques explicites, et en insistant sur la mise en œuvre des intentions stratégiques (Koenig, 1990). Les tenants de ce courant, en mettant aussi en avant la dimension stratégique des activités quotidiennes (concept de gestion stratégique, par opposition à une planification stratégique conçue comme un exercice à effectuer à certains intervalles), ont conscience d'une nécessaire adéquation entre stratégie et fonctions opérationnelles de l'entreprise. Mais les problèmes précis d'articulation entre stratégie, d'une part, marketing, finance et relations humaines, d'autre part, ne sont pas souvent examinés de façon explicite par ces auteurs. Ils préfèrent les aborder 


\section{FIGURE 1}

Les principales étapes de la planification stratégique et les apports des stratégies du marketing à chacune des ces étapes

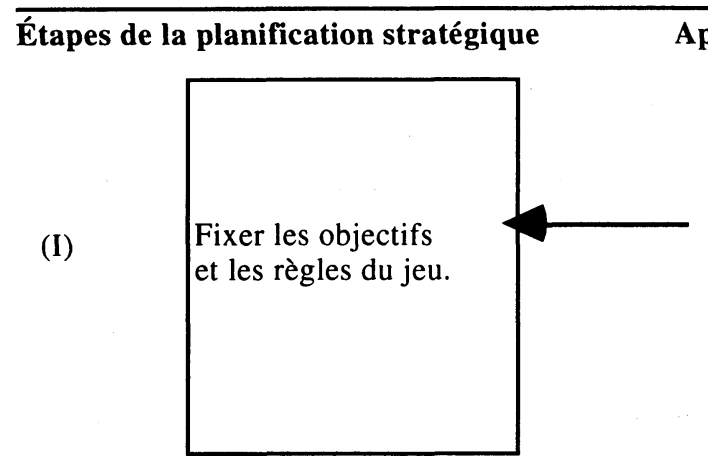

Apports des stratégies marketing

Contribuent à faire définir la mission de manière plus large qu'en termes de produits fabriqués.

Rôle possible au niveau des « règles de conduite » (respect des droits des consommateurs).

Analyser les lignes de force et de faiblesse de l'entreprise.

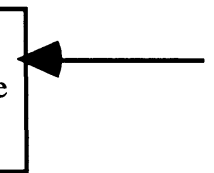

(III) concurrentiel des prochaines années.
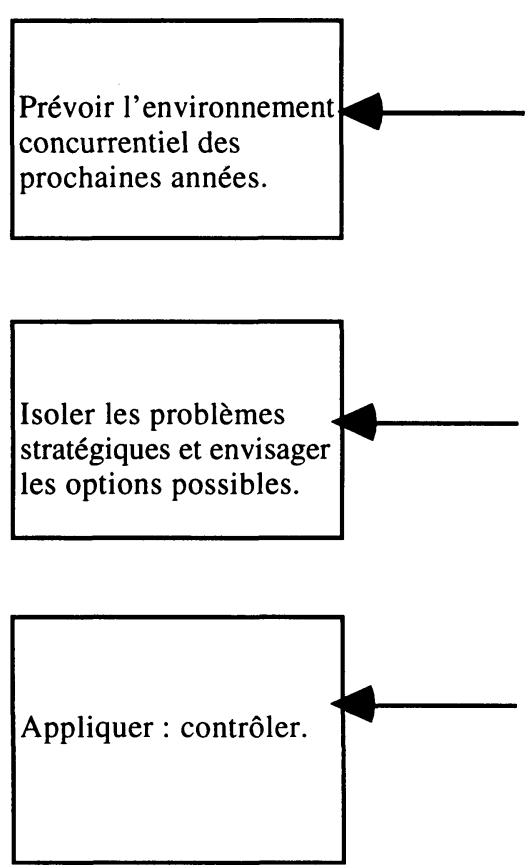

Participation du marketing au diagnostic d'ensemble de l'entreprise.

Rôle fondamental au niveau de la prévision des marchés futurs, de la concurrence, des structures de la distribution.

Peuvent ajouter une précision "marketing " aux options stratégiques choisies (exemples : la commandite, le système de franchise).

Contribuent, pour une très large part, au succès des options stratégiques, en les mettant en œuvre par le biais du plan du marketing.

Source : B. Saporta (1986). 
par le biais des structures, en restant le plus souvent à un degré assez élevé de généralité.

\subsection{Les angles d'approche de la littérature marketing}

Les spécialistes du marketing ont tenté à plusieurs reprises des rapprochements entre leur discipline et la stratégie, au point que les frontières qui les séparent sont parfois difficiles à définir. Sans nous engager dans un débat qui partage les auteurs, on évoquera brièvement quelques contributions utiles pour nos développements ultérieurs.

Le terme de marketing stratégique semble au premier abord assez prometteur au regard du thème qui nous intéresse. Mais les tenants de cette approche traitent moins de l'articulation précise entre les deux disciplines (entre plan stratégique et plan marketing, par exemple) que de la nécessité de distinguer au sein même du marketing deux niveaux de préoccupations: assurer à l'entreprise un avantage concurrentiel défendable grâce à une démarche d'analyse systématique des besoins du marché et un choix raisonné de cibles et de positionnements (marketing stratégique); mettre en action des programmes de distribution, prix, vente et communication destinés à faire connaître et à valoriser les choix précédents auprès des cibles retenues (marketing opérationnel ou «bras armé » du marketing) (Lambin, 1989). D'autres spécialistes, en opposant dans le même esprit marketing stratégique et marketing management, soulignent que seul le premier examine de manière privilégiée la façon dont l'entreprise agit face à la concurrence; les outils utilisés sont alors ceux de l'analyse stratégique classique, conjointement avec les choix de segmentation des marchés et de positionnement, placés à juste titre très en amont dans l'ordre des préoccupations d'un responsable marketing (Dubois et Jolibert, 1992, p. 221 et suiv.).

Ce souci d'élever le marketing au rang des préoccupations stratégiques de l'entreprise devrait normalement coïncider avec une plus grande utilisation des compétences marketing dans le pilotage stratégique, notamment au sein des groupes. Un certain nombre de contributions apportent à cet égard des conclusions mitigées. Au regard des choix stratégiques «corporatifs » (dans quels métiers devons-nous travailler? ), une enquête effectuée en 1989 aux États-Unis auprès de 243 grandes entreprises montre que la fonction marketing jouait un rôle plutôt mineur dans les tâches de planification des états-majors (Raymond et Barksdale, 1989). Une raison avancée à cet état de choses est l'incapacité des responsables marketing à prendre réellement en considération les demandes des autres «parties prenantes» que les consommateurs, comme par exemple les actionnaires, les salariés, les hommes politiques, le public en général (Aaby et McGann, 1989). Au regard des choix stratégiques «d'affaire» 
(comment affronter la concurrence dans un métier donné ? ), le rôle de la fonction marketing est beaucoup plus évident, au point de se confondre parfois avec la problématique stratégique elle-même. On en prendra comme exemple les nombreux articles relevant l'intérêt des stratégies marketing offensives d'augmentation de part de marché pour les petits compétiteurs; l'un des plus récents propose au lecteur une grille d'évaluation multicritère de ces stratégies (Stasch et Ward, 1989).

Le thème est abordé de manière plus directe lorsqu'on met en relief $l e$ rôle du marketing dans la mise en cuvre des choix stratégiques. Michael Porter ne manquait pas dès le départ de souligner que ses stratégies « génériques » ne pouvaient réussir qu' accompagnées d'un marketing approprié (émission de «signaux de valeur» pour la stratégie de différenciation). Un essai assez complet a été tenté pour dégager les implications marketing de la typologie stratégique de Miles et Snow (McDaniel et Kolari, 1987). On examinera ici de plus près une contribution qui combine les typologies de Porter et de Miles et Snow, pour aboutir à des stratégies «hybrides », et suggérer quels types d'organisations et de programmes marketing sont les plus susceptibles de contribuer au «succès ${ }^{1}$ » de ces stratégies (Walker et Ruekert, 1987).

Nous sommes ici en présence d'un véritable «agenda de recherche» qui, selon nous, n'a reçu à ce jour aucune vérification empirique précise (les auteurs citent des traces d' "évidences» dans la littérature). Il est cependant douteux qu'il puisse s'appliquer en l'état dans un contexte entrepreneurial. Il a en effet surtout pour objectif de jeter les bases d'un «marketing contingent» au sein de groupes diversifiés. En outre, les stratégies visées par l'étude sont plutôt des «postures» ou des «positionnements stratégiques » que de véritables projets entrepreneuriaux, catégorie qui nous semblera plus adaptée dans nos développements ultérieurs. En adoptant cet agenda en milieu PME - où les stratégies sont rarement explicites -, on court le risque d'inverser la question de recherche : les comportements marketing, plutôt que d'être perçus comme une condition de la bonne exécution d'une stratégie, peuvent alors être considérés comme des indicateurs ou révélateurs d'un positionnement stratégique difficile à déceler par observation directe ${ }^{2}$.

1. Les auteurs soulignent justement que les indicateurs de performance-mesures du succès - doivent différer selon le positionnement stratégique adopté. Le succès d'un « prospecteur » se mesurera à l'aune de sa créativité (taux de lancement de nouveaux produits) et/ou de son efficacité (progrès en parts de marché), tandis que celui d'un « défensif à coûts bas " se vérifiera à l'aide de critères d'efficience (ROI).

2. Pour un essai très complet dans cette direction, voir d'Amboise et Fortin (1989). Les comportements marketing ne sont toutefois, dans cette étude, qu'un indicateur parmi d'autres d'un positionnement stratégique. 
TABLEAU 1

Programmes marketing susceptibles d'être associés au succès de trois stratégies génériques «hybrides» combinant les typologies de Porter et de Miles et Snow

\begin{tabular}{|c|c|c|c|}
\hline $\begin{array}{l}\text { Typologie } \\
\text { stratégique } \\
\text { Éléments du } \\
\text { programme } \\
\text { marketing }\end{array}$ & «Prospecteur» & $\begin{array}{l}\text { «Défensif } \\
\text { différencié » }\end{array}$ & $\begin{array}{l}\text { «Défensif } \\
\text { à coûts bas » }\end{array}$ \\
\hline $\begin{array}{l}\text { Gammes de } \\
\text { produits }\end{array}$ & $\begin{array}{l}\text { larges, } \\
\text { techniquement } \\
\text { sophistiquées }\end{array}$ & $\begin{array}{l}\text { étroites, qualité } \\
\text { relativement élevée }\end{array}$ & $\begin{array}{l}\text { étroites, } \\
\text { techniquement peu } \\
\text { sophistiquées }\end{array}$ \\
\hline Service & $\begin{array}{l}\text { niveau relativement } \\
\text { élevé }\end{array}$ & $\begin{array}{l}\text { niveau relativement } \\
\text { élevé }\end{array}$ & $\begin{array}{l}\text { niveau relativement } \\
\text { bas }\end{array}$ \\
\hline Prix & relativement élevés & relativements élevés & relativement bas \\
\hline $\begin{array}{l}\text { Contrôle des canaux } \\
\text { de distribution }\end{array}$ & relativement faible & relativement élevé & relativement élevé \\
\hline $\begin{array}{l}\text { Dépenses de } \\
\text { publicité et de } \\
\text { promotion }\end{array}$ & relativement élevées & moyennes & relativement faibles \\
\hline $\begin{array}{l}\text { Dépenses en force } \\
\text { de vente }\end{array}$ & moyennes & relativement élevées & moyennes \\
\hline
\end{tabular}

Source : D'après Walker et Ruekert (1987).

\section{Plan marketing et choix stratégiques entrepreneuriaux: une cohérence qui va de soi?}

La recherche d'une cohérence entre stratégie et marketing semble au premier abord plus aisée pour l'entrepreneur, ce qui explique peut-être l'absence de traitement explicite de cette question dans la littérature ${ }^{3}$. Un examen de cas réels montre toutefois que, dans la pratique, des dysfonctionnements existent, avec des conséquences gênantes pour la survie de l'entreprise, sollicitant par là même l'attention du chercheur.

\subsection{Une cohérence au premier abord plus facile à obtenir...}

On peut citer en faveur de cette hypothèse un certain nombre de caractéristiques du modèle entrepreneurial.

3. Les spécialistes de la création d'entreprise, par exemple, l'abordent à peine, notamment lorsqu'ils présentent les principes de rédaction du plan d'affaires. Pour une synthèse récente et un effort méritoire d'intégration du marketing à la stratégie dans le plan d'affaires, voir Belley, Dussault et Lorrain (1991). 


\subsubsection{La concentration des pouvoirs en une seule et même personne}

La prééminence du système de valeurs de l'entrepreneur dans le pilotage stratégique de son entreprise a été maintes fois soulignée. Tout dépend, en fait, des motivations qui ont poussé le dirigeant à créer son affaire, ou à la garder. Parmi ces motivations, certaines ne sont pas toujours compatibles avec un développement sain de l'entreprise (comme, par exemple, le désir de se réaliser personnellement; créer une source d'emplois pour sa famille; se ménager du temps libre pour d'autres activités; créer une organisation dont on est le seul maître, sans interférences de quiconque, etc. ${ }^{4}$ ). La coexistence chez un même individu d'objectifs personnels et d'objectifs assignés à l'entreprise est en soi une source de tension psychologique pour le dirigeant, qui a pu être catégorisé selon la manière dont il résout ce type de contradiction (Laufer, 1975). Elle représente, en outre, une gêne possible dans le processus de formulation des stratégies (des choix stratégiques, guidés en apparence par l'intérêt de l'entreprise, peuvent être le reflet de préoccupations beaucoup plus personnelles, comme celle de garder le contrôle de son affaire).

Malgré tout, cette unicité du pouvoir décisionnel représente un avantage lorsque le moment est venu de mobiliser des activités opérationnelles - comme la mise en place d'un plan d'action marketing - au service d'une stratégie. En premier lieu, le dirigeant n'est pas tenu d'expliquer sa stratégie à un responsable commercial, puisque c'est lui seul qui effectue la synthèse entre ce qui relève de son "grand dessein » et ce qui relève plutôt de sa mise en application. Ensuite, et on arrive alors à l'avantage si souvent cité de flexibilité des petites organisations, il a tout loisir, grâce à un système personnel d'information plus performant qu'on ne le croit généralement, d'adapter non pas sa stratégie, mais les conditions de son application, aux changements de l'environnement ${ }^{5}$.

4. La diversité de ces motivations a donné lieu à de nombreuses typologies d'entrepreneurs, dont la description dépasse le cadre de cette étude, mais dont l'existence ne peut être ignorée dans notre proposition de recherche empirique. Pour une analyse critique de ces typologies, accompagnée d'une classification originale faisant l'économie du mot « entrepreneur », voir Hornaday (1990).

5. Plutôt qu'une reformulation continuelle de ses choix stratégiques, la flexibilité des entreprises performantes consisterait en une adaptation fine et incessante d'une stratégie globale dont les grandes lignes resteraient inchangées (Porter, 1991, p. 110). 


\subsubsection{L'existence d'une "vision entrepreneuriale"}

Le concept de vision, qui fait l'objet d'une attention récente dans la littérature stratégique, a été étendu depuis peu à ce qui devrait constituer son domaine d'élection, celui de l'entrepreneur (Carrière, 1990; Filion, 1991). Ce dernier, lorsqu'il mérite son nom, serait doté d'une aptitude particulière à projeter et à visualiser dans l'avenir la place qu'il veut occuper sur un marché, ainsi qu'une image précise de l'entreprise dont il a besoin pour accomplir cet objectif. À ces facultés de «visionnaire» s'ajouteraient celles de «leader », lesquelles lui permettraient de faire comprendre et de partager cette vision avec ses proches, y compris ses collaborateurs (Filion, 1991). On est ici en présence d'un trait pouvant expliquer pourquoi les plans stratégiques, explicites ou implicites, sont plus facilement compris et exécutés dans une organisation de type entrepreneurial, mais dont la taille exige désormais un certain degré de délégation au regard de la mise en application des stratégies. On aboutit alors à un type de planification stratégique «dominée par le leader», considérée par certains observateurs comme l'un des modes les plus efficaces que l'on puisse trouver dans ce domaine (Allaire et Firsirotu, 1990, p. 24).

\subsubsection{La pratique d'un mode particulier de planification stratégique par l'entrepreneur}

Nous ne reviendrons pas ici en détail sur la question de savoir si les dirigeants de PME ont ou non une activité de planification stratégique, de quel type elle est et en quoi ces pratiques sont associées à la performance (pour une synthèse complète, voir d'Amboise et Bakanibona, 1990). On conclut le plus souvent que dans les petites entreprises la planification stratégique est un exercice plutôt rare et généralement peu formalisé: c'est ainsi qu'une enquête effectuée auprès de 115 entreprises américaines de moins de 100 salariés a révélé que seulement 24 d'entre elles pratiquaient un type de planification stratégique à la fois formalisé (rédaction d'un plan écrit) et prenant en compte des données assez générales d'environnement (extensive formal planning) (Schrader, Mulford et Blackburn, 1989). Nous sommes donc en présence d'un comportement planificateur en grande partie intuitif, informel, axé plus sur la réponse à une occasion favorable ou à une menace immédiate qu'à un processus systématique et raisonné de résolution d'un problème stratégique. Or, ce comportement facilite la prise en considération des contraintes opérationnelles (très présentes dans l'esprit de beaucoup d'entrepreneurs) au moment même où les choix stratégiques sont pris (encore une fois, ces processus, lorsqu'ils existent, mériteraient d'être étudiés de plus près). 


\section{2. ... mais dont les dysfonctionnements restent possibles (sinon probables)}

Dans la pratique, comment s'effectue la liaison entre stratégie et marketing chez un entrepreneur? La validité externe des deux cas réels exposés ci-après demeure certes limitée, mais leur examen n'en constitue pas moins un indice précieux de situations qui peuvent se révéler relativement fréquentes.

\subsubsection{Un exemple de confusion entre décision marketing et décision stratégique (cas d'une PME en régime de croisière)}

Très souvent sont considérées comme exclusivement d'ordre marketing des décisions qui ont en réalité une nature stratégique. Il en résulte des dysfonctionnements, dont le cas ci-après donne une illustration ${ }^{6}$ :

«Nous ne sommes qu'une modeste PME (45 salariés), et pourtant nous vivons en grande partie de contrats décrochés à l'étranger », reconnaît le p.-d.g. de cette entreprise spécialisée dans les travaux d'ingénierie mécanique (remise en état de turbines, vannes et champs pétrolifères).

Cette activité de chantiers civils en Afrique et au Moyen-Orient, ce dirigeant la connaît bien pour l'avoir longtemps pratiquée dans une grande entreprise, avant de reprendre en 1982 une affaire en règlement judiciaire. Le démarrage sera extrêmement rapide mais ne le satisfait pas entièrement, car le chiffre d'affaires repose sur des appels d'offres lourds et aléatoires faisant peser sur la jeune société un risque jugé insupportable. Par ailleurs, le suivi des chantiers immobilise loin de ses bases une bonne partie du personnel qualifié (ingénieurs d'affaires), qui souhaiterait peut-être un mode de vie plus propice à la vie familiale...

En vue de remédier à cette situation de risque, un autre type d'activité a été privilégié, cette fois centré sur l'Hexagone: l'obtention de contrats de maintenance auprès de groupes industriels désireux de se débarrasser en tout ou en partie de cette activité de plus en plus coûteuse à conserver en propre. On a donc procédé au recrutement d'un ingénieur technico-commercial chargé de prospecter systématiquement ce nouveau type d' «affaires». D'un certain point de vue, on a donc considéré comme relevant essentiellement du «commercial » une option de rééquilibrage des activités qui a un caractère fondamentalement stratégique. Le bien-fondé de cette option paraît incontestable, mais la démarche adoptée comporte deux inconvénients.

6. L'un des auteurs anime des programmes expérimentaux de formation au management stratégique de dirigeants de PME, d'où sont tirés les cas développés dans cette étude. 
La responsabilité de la nouvelle «activité » risque d'être placée trop bas dans l'organigramme. On la confie à l'ingénieur chargé de la "prospection », mais son rôle n'est-il pas plutôt d'exécuter sur le plan commercial, des directives émanant d'un échelon hiérarchique supérieur? Cette anomalie sur le plan organisationnel sera encore plus gênante si des difficultés non prévues au départ surgissent en cours de route, comme cela s'est produit.

Une prise en considération insuffisante de l'aspect stratégique de l'option a en effet empêché l'entreprise d'en cerner, à temps, les contours et les contraintes. Par exemple, les compétences requises par les deux «métiers» (chantiers d'ingénierie et maintenance) ne sont pas exactement les mêmes. L'activité d'ingénierie nécessite une grande compétence des ingénieurs qui définisssent le process, alors que les techniciens qui réalisent le chantier n'ont qu'une compétence moyenne. Le problème est inverse pour la maintenance, qui exige des techniciens de très haut calibre (la maintenance assurée par l'entreprise s'assimile au service du constructeur). La qualification et l'expérience de ces techniciens ont donc été perçues - mais un peu tardivement - comme une contrainte majeure pour progresser dans cette direction. Le nombre des spécialistes dans l'entreprise étant réduit, il a fallu en embaucher un certain nombre de nouveaux, en les prenant déjà formés, au prix de salaires et de conditions de travail attrayants, chez les principaux constructeurs de turbo-machines...

\subsubsection{Un exemple de décision stratégique prise à bon escient, mais sans anticiper suffisamment ses contraintes marketing (cas d'une PME en démarrage)}

Une autre manifestation d'inadéquation entre marketing et stratégie consiste pour un dirigeant à ne pas se rendre compte des répercussions commerciales de décisions stratégiques en soi fondées. Par exemple, une entreprise de soustraitance se lancera dans la fabrication de produits propres, sans suffisamment prévoir les moyens commerciaux qu'exige cette décision stratégique par excellence, généralement prise pour échapper à une situation de dépendance excessive. Le cas ci-après, décrivant une situation d'entreprise en démarrage, en fournit une autre illustration.

Cette petite entreprise, créée au début de 1987, constituait un cas intéressant d'entreprise en démarrage. Son domaine de spécialité était la conception, la fabrication et la commercialisation d'instruments chirurgicaux de haute précision destinés à la chirurgie de l'œil, des zones ORL et à la microchirurgie en général. Étroitement associé au créateur se trouvait un technicien ayant travaillé de longues années chez le principal concurrent et connaissant parfaitement tant les contraintes techniques de fabrication que celles relatives 
à la clientèle (chirurgiens). Le p.-d.g. et créateur, quant à lui issu de la grande distribution, possédait une longue expérience de la conduite et de la gestion de projets. Son ouverture d'esprit, son sens de la communication et la maîtrise des techniques du marketing devaient apporter un ton nouveau dans une profession quelque peu figée par les habitudes.

L'entreprise connut, cependant, dès l'année 1988, des problèmes graves de démarrage, ayant provoqué une dégradation inquiétante de ses structures financières. Le chiffre d'affaires prévu pour les deux premières années d'exercice n'avait pu se développer, à cause de retards de mise en place commerciale, mais aussi de l'inertie d'un marché caractérisé par des positions acquises de longue date. Au début de l'année 1989, dans l'impossibilité de réunir les fonds nécessaires à un renflouement des stocks financiers, le p.-d.g. dut se résigner à abandonner la partie et à déposer son bilan...

Un diagnostic de ce cas, étayé par une documentation assez complète et des entretiens auprès des principaux acteurs, permit de dégager les deux conclusions suivantes.

1. Il y avait, semble-t-il, une place à prendre pour la nouvelle entreprise dans le marché français (et des pays limitrophes) de l'instrumentation médicale (la décision stratégique de lancement était donc en assez grande partie fondée).

En effet, le marché était relativement ouvert à un nouveau venu :

- La taille du marché - dans les spécialités concernées - se situait aux alentours de 150 millions de francs: les chiffres d'affaires escomptés par la nouvelle n'en représentant qu'une part minime, cela devait contribuer à retarder les réactions de la concurrence.

- Les sous-marchés respectifs étaient dominés par un très petit nombre d'acteurs, ce qui conduisait inévitablement à un «impérialisme commercial»: prix élevés, politique commerciale et de services laxiste, donc une source d'occasions d'affaires pour un nouveau venu. L'accroissement consécutif des importations ne pouvait entièrement combler ce vide, en raison de prix élevés et de délais de livraison importants.

- Les voyages de prospection du p.-d.g. dans les pays voisins avaient montré des carences certaines dans les politiques d'exportation des fabricants français (ventes directes court-circuitant les revendeurs locaux, problèmes d'exclusivité, etc.), laissant la place à des stratégies claires de distribution sur le plan internationnal. 
Face à ces circonstances opportunes, les propositions de la nouvelle PME comblaient un vide certain :

- Une qualité d'instruments au moins égale à celle des concurrents français, et supérieure à celle des produits importés, en raison de sa spécialisation sur la seule microchirurgie.

- Un service exceptionnel, puisque la PME annonçait des délais de livraison très courts, une maintenance et une réparation rapide, une volonté d'écoute des chirurgiens sur le plan des innovations, tous facteurs facilités par une implantation industrielle sur le territoire français.

- Des prix plus bas que ceux demandés par l'ensemble de la concurrence, accompagnés par ailleurs d'une politique de remises pour achats importants inexistante jusque-là dans la profession.

- Une gamme très complète, grâce au recours au négoce, par le biais d'un artisan qui confia à la PME la commercialisation d'une grande partie de ses instruments, d'une excellente qualité de fabrication.

- Un style de communication moderne et dynamique, tranchant avec le conservatisme de la concurrence.

2. Comment expliquer le retard du chiffre d'affaires pris dans les premières années d'exercice (et les pertes consécutives à ce retard)?

Le dirigeant-créateur, en dépit de son expérience dans le domaine commercial (laquelle n'était peut-être pas directement transposable dans le nouveau métier qu'il s'était choisi), a commis, semble-t-il, trois erreurs majeures dans le domaine du « suivi marketing » du projet:

- Les moyens commerciaux susceptibles de faire démarrer le chiffre d'affaires ne furent mis en place qu'avec retard. Les deux éléments à signaler à cet égard sont, d'une part, le catalogue, qui mit beaucoup plus de temps que prévu à être mis au point, et surtout la très grande difficulté, non prévue par le créateur, à recruter un réseau de vendeurs exclusifs dans une spécialité très étroite et où les compétences étaient déjà utilisées par la concurrence. L'idée d'avoir recours à un revendeur, auquel le p.-d.g. songea en cours de route, fut abandonnée, car cela l'aurait obligé à renoncer aux avantages stratégiques par rapport à la concurrence décrits plus hauts.

- Le dirigeant avait, par ailleurs, sous-estimé le coût des investissements commerciaux de départ. Le catalogue ophtalmologique, à lui seul, coûta $600000 \mathrm{~F}$, soit dix fois plus que les estimations du 
plan d'affaires initial! Si on y ajoute le coût des valises des représentants, ainsi que de certains autres postes, il s'agit de véritables investissements qu'il aurait fallu chiffrer dès le départ, en modifiant le plan de financement initial en conséquence.

- Et surtout, il n'avait pas prévu les facteurs d'inertie du marché de l'instrumentation médicale. Cette confiance difficile à acquérir, avec une concurrence déjà installée, liée aux caractéristiques du marché (circuits d'approvisionnement lourds et complexes en milieu hospitalier, poids des habitudes) fut signalée à plusieurs reprises dans une étude commandée à un cabinet pendant la phase de démarrage, mais apparemment ce point capital ne fut pas suffisamment intégré dans les stratégies du dirigeant...

Les dysfonctionnements assez sérieux que décrivent ces deux cas illustrent les limites des conditions favorables de cohérence stratégie/marketing qui viennent d'être exposées. L'explication de ces dysfontionnements tient en grande partie à l'extrême personnalisation de la décision autour du propriétaire-dirigeant. Cette centralisation a deux conséquences. D'une part, elle conduit à un manque de recul - pourtant nécessaire à la réflexion stratégique - causé par une implication trop grande de l'entrepreneur dans les activités opérationnelles. D'autre part, elle constitue un filtre qui modèle la perception de l'environnement selon la personnalité du dirigeant (traitement de l'information, attitude face au risque, capacité d'anticipation) et de ses valeurs (responsabilité sociale, indépendance, pouvoir) (Bamberger, 1982). Et ce filtre a une influence d'autant plus forte que les PME manquent généralement de système d'information formalisé (Debrinay, 1990).

\section{Vers une approche des pratiques des dirigeants de PME}

Les développements précédents posent le problème de la recherche de cohérence entre stratégie et marketing pour un dirigeant de petite entreprise et tracent les grandes lignes de leurs spécificités.

Pour proposer aux responsables des modèles plus adaptés, il y a sans nul doute une nécessité pour le chercheur de se tourner vers le potentiel d'investigations que constitue l'observation sur le terrain de ces comportements d'intégration.

Les points de repère fournis par la littérature stratégique et marketing constituent un cadre pour aborder cette question, au sein duquel des choix doivent être effectués. 


\subsection{Comment l'entrepreneur concilie-t-il en pratique ses choix stratégiques (grand dessein) et son plan marketing?}

Pour que l'étude de situations concrètes puisse fournir des éléments d'observation et d'analyse, il convient d'envisager deux types d'interrogations:

1. Comment le problème de la cohérence entre la stratégie et le marketing doit-il être abordé?

2. Comment cette cohérence peut-elle être observée à travers le mode si particulier du processus de planification de l'entrepreneur?

\subsubsection{L'adaptation du cadre conceptuel}

Les conclusions des auteurs convergent sur l'importance des activités opérationnelles comme facteur de performance pour les PME, notamment lorsque ces activités sont en adéquation avec les choix stratégiques de l'entreprise.

Le problème pourrait, selon nous, être abordé à partir de l'analyse de la cohérence entre l'orientation stratégique de l'entreprise et la nature des activités opérationnelles d'ordre marketing considérées comme critiques dans la mise en cuvre de ces choix.

Cette démarche suppose une double réflexion, d'une part, sur la typologie des choix stratégiques des PME qui doit être prise en compte et, d'autre part, sur la nature des activités opérationnelles qui doivent être considérées comme critiques pour chaque stratégie générique.

Il s'agirait alors de construire un cadre conceptuel de référence qui permettrait de préciser, en fonction d'un projet de nature stratégique, les caractéristiques que le plan marketing devrait posséder pour que l'intégration stratégie/ marketing soit réelle. Les travaux de Walker et Ruekert (voir plus loin, figure 2) s'inspirent de ce principe, mais ils nous semblent présenter des limites puisqu'ils n'ont pas fait l'objet de vérification empirique précise et qu'ils s'appuient sur des stratégies génériques qui ne nous apparaissent pas forcément adaptées aux situations les plus fréquentes des PME (cas de l'option «défensif à coûts bas », par exemple). De fait, il s'agit surtout - pour une petite ou moyenne entreprise - de formuler des choix destinés à maximiser ses chances de survie en tentant de s'intégrer dans son environnement. Deux axes clés apparaissent essentiels pour analyser cette intégration: le degré de vulnérabilité induit par le type d'activité et le degré de dépendance induit par le type d'acteur (Julien et Marchesnay, 1988). 
À cet égard, Koenig distingue trois types de politiques relationnelles pour conduire le pilotage de l'entreprise dans un espace stratégique où interviennent non seulement les concurrents, mais aussi les autres acteurs indispensables à l'activité de l'entreprise (premier axe clé: le degré de dépendance relatif au type d'acteur). Il s'agit de l'affrontement, de l'évitement et de la coopération. Mais ces différentes politiques relationnelles ne correspondent pas toutes de la même façon au cas des PME. Ainsi, ces derniers ne peuvent pas envisager une stratégie d'affrontement: le rapport de force avec les autres acteurs de l'environnement leur est en général défavorable.

C'est pourquoi il paraît pertinent de nuancer cette typologie par l'idée que la PME doit avant tout formuler des choix destinés à maximiser ses chances de survie (deuxième axe clé: le degré de vulnérabilité). Saporta propose ainsi une classification séquentielle des options de la PME et envisage trois axes: l'innovation, le renforcement et le redéploiement.

La complémentarité de ces deux travaux suscite une réflexion sur les recoupements éventuels des situations qu'ils envisagent. Parmi les politiques relationnelles définies par Koenig, quelles sont celles qui correspondent aux options stratégiques de la PME, telles que les présente Saporta ? Construit à partir des commentaires de chaque auteur sur les objectifs relatifs à chaque stratégie, le tableau présenté montre que l'on peut finalement isoler deux types de comportement stratégique plus susceptibles de convenir aux PME: la stratégie de distinction, d'une part, qui regroupe les options d'innovation et de renforcement, et la stratégie de croissance, d'autre part? ${ }^{7}$.

Il s'agit ensuite d'expliciter les incidences de l'option stratégique retenue sur les activités opérationnelles relevant du domaine marketing. Il serait prématuré de proposer, dans le cadre de cette réflexion, une liste exhaustive de fonctions critiques associées à chaque option stratégique. Nous nous contenterons donc d'illustrer notre propos par deux exemples.

Une option stratégique de croissance peut, par exemple, correspondre à une volonté de la PME d'échapper aux facteurs de vulnérabilité liés à son activité en se redéployant sur un marché nouveau pour elle, mais cependant en synergie avec son activité actuelle (option de redéploiement/Saporta; politique de coopération de type spécialisation/Koenig). L'entreprise recherche alors un supplément de chiffre d'affaires et souhaite bénéficier d'un avantage stratégique conféré par la taille. La fonction marketing doit donc prioritairement mettre en ouvre une stratégie s'appuyant sur une politique marketing permettant d'introduire l'entreprise auprès d'une nouvelle clientèle (par le biais d'une modification du positionnement ou de la distribution).

7. Si l'on envisage l'option de redéploiement comme un développement de l'entreprise dans les activités dont elle maîtrise déjà l'essentiel du métier. 


\section{TABLEAU 2}

\section{Une typologie des politiques relationnelles dans le cas des options stratégiques de la PME}

\begin{tabular}{|c|c|c|c|}
\hline & \multicolumn{3}{|c|}{ Politiques relationnelles (Koenig) } \\
\hline $\begin{array}{l}\text { Options stratégiques } \\
\text { de la PME (Saporta) }\end{array}$ & Affrontement & Évitement & Coopération \\
\hline Innovation & \multirow[t]{2}{*}{$\begin{array}{l}\text { Domination par les } \\
\text { coûts ; modalités } \\
\text { d'accès aux } \\
\text { ressources ; }\end{array}$} & $\begin{array}{l}\text { «Porter le combat } \\
\text { sur un terrain } \\
\text { propice aux PME » } \\
\text { (Saporta) }\end{array}$ & \\
\hline $\begin{array}{l}\text { Renforcement : } \\
\text { Spécialisation } \\
\text { étroite } \\
\text { Gestion de la } \\
\text { dépendance }\end{array}$ & & $\begin{array}{l}\text { «Une protection } \\
\text { contre la } \\
\text { concurrence des } \\
\text { grandes } \\
\text { entreprises » } \\
\\
\text { «Savoir se rendre } \\
\text { indispensable» } \\
\text { (Saporta) }\end{array}$ & $\begin{array}{l}\text { «L'individualisme } \\
\text { des dirigeants de } \\
\text { PME est un frein } \\
\text { fréquent aux } \\
\text { stratégies de } \\
\text { coopération » } \\
\text { (Saporta) }\end{array}$ \\
\hline $\begin{array}{l}\text { Redéploiement : } \\
\text { Diversification } \\
\text { domestique }\end{array}$ & $\begin{array}{l}\text { Dans tous les cas, le } \\
\text { rapport des forces } \\
\text { est défavorable à la } \\
\text { PME. }\end{array}$ & & $\begin{array}{l}\text { Développer une } \\
\text { activité nouvelle sur } \\
\text { le marché français. } \\
\text { Rechercher un } \\
\text { supplément de } \\
\text { chiffre d'affaires sur } \\
\text { un marché étranger. } \\
\text { (Saporta) }\end{array}$ \\
\hline & & $\begin{array}{l}\text { Option de } \\
\text { distinction «Éviter } \\
\text { l'affrontement } \\
\text { stratégique » } \\
\text { (Koenig) }\end{array}$ & $\begin{array}{l}\text { Manœuvre de } \\
\text { croissance : } \\
\text { bénéficier de } \\
\text { l'avantage conféré } \\
\text { par la taille (Koenig) }\end{array}$ \\
\hline
\end{tabular}

Par exemple, dans le cas d'un début d'activité à l'exportation (redéploiement par l'internationalisation), les fonctions opérationnelles critiques d'ordre marketing pourraient être les suivantes (Walker et Ruekert, 1987, Léo et al., 1989):

- mise en place d'une structure spécialisée, recherche d'informations sur le marché ;

- mise en place de procédures de recouvrement de créances;

- mise en place d'un réseau local de distribution; 
- adaptation du produit aux attentes locales (prix, caractéristiques techniques, etc.).

Une autre option stratégique peut, par contre, correspondre à une volonté de l'entreprise d'éviter «un choc frontal avec la concurrence » en concevant une offre qui ne puisse être directement comparée à aucune autre, cela grâce à un caractère innovant ou une compétence rendue crédible par une spécialisation de la PME (politique d'évitement / Koenig; option d'innovation/Saporta). La perception par le client de la différence que l'entreprise souhaite promouvoir constitue alors l'un des facteurs clés de la réussite de ce type de stratégie. Le rôle du marketing consiste ici à agir sur les signaux de valeur (emballage, communication, etc.) de manière à ce que le client puisse évaluer correctement le supplément de valeur procuré par l'offre.

Le cas de l'exemple cité dans la première partie (entreprise spécialisée dans la conception fabrication et commercialisation d'instruments de chirurgie de haute précision) correspond bien à ce type de choix stratégique. Les fonctions opérationnelles critiques d'ordre marketing pourraient être notamment (Walker et Ruekert, 1987):

- signalisation de la valeur;

- politique de prix : fermeté tarifaire;

- importance du budget R-D;

- qualité des prestations de service;

- adaptation de la force de vente (compétence, extension).

Or, le diagnostic du cas montre que le retard pris sur la constitution du catalogue et la difficulté rencontrée pour mettre en place un réseau de vendeurs compétents peuvent expliquer en partie le retard du chiffre d'affaires dans les premières années. Ces erreurs commises par le dirigeant-créateur peuvent être interprétées comme une incohérence entre les choix stratégiques et le suivi des fonctions critiques opérationnelles d'ordre marketing.

Une telle approche se heurte toutefois aux spécificités du processus de décision entrepreneurial : en effet, l'unicité du pouvoir décisionnel ne favorise pas l'explicitation des décisions qui relèvent des choix stratégiques («grand dessein ») ni de celles qui correspondent à sa mise en application.

De manière générale (Stratégor, 1988), on distingue les situations de décision émergente. Leur particularité la plus notable est qu'elle ne donne pas lieu à une stratégie voulue explicite (prévue) la plus proche des doctrines traditionnelles (et donc occurrente) en réaction à une « surprise stratégique». Au sein des PME, ces situations vont engendrer deux types de comportements : l'entrepreneur peut en effet se conduire comme un «preneur de décision » ou 
un «résolveur de problème». Dans leurs travaux consacrés aux processus et procédures stratégiques de ce type d'entreprises, Chicha, Julien et Marchesnay montrent que les dirigeants se révèlent finalement plus «proactifs » que « réactifs », contrairement à ce qu'un courant assez fort de la littérature pouvait laisser supposer. La synthèse entre les décisions d'ordres stratégique et opérationnel se produit ainsi à l'issue d'un processus qui reste, de manière générale, mal connu.

FIGURE 2

L'intégration des choix stratégiques entrepreneuriaux et du plan marketing : un cadre conceptuel d'analyse

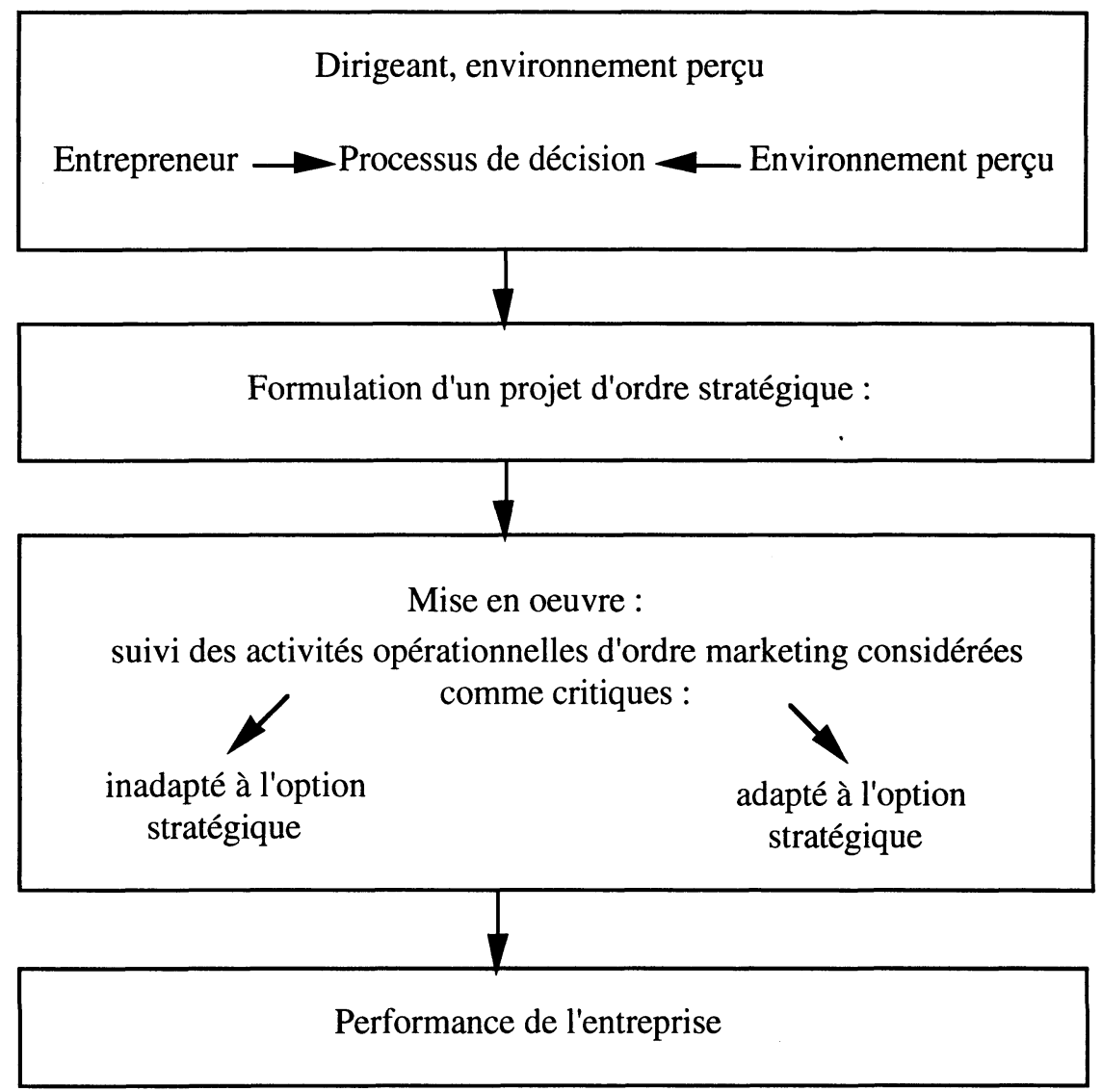


Le cadre d'analyse que nous proposons ne cherche pas à explorer une fois de plus le processus de décision entrepreneurial. Il le considère plutôt comme une sorte de «boîte noire» qui engendre un projet de nature stratégique. Nous nous intéressons ensuite à la façon dont ce projet est mis en œuvre.

\subsection{Proposition d'un cadre d'analyse}

Ce cadre (figure 2) est centré sur la formulation puis la mise en œuvre, par le dirigeant, d'un projet de nature stratégique.

Ce projet doit pouvoir être mis en relation avec l'une des options stratégiques identifiées grâce à la typologie dont nous venons de décrire les principes de construction. Le recours au concept de projet présente l'intérêt d'éviter deux écueils. D'une part, le choix de raisonner sur une population de projets plutôt que d'entreprises permet de mieux cerner le cadre concerné par les décisions du dirigeant et limite la contrainte de l'hétérogénéité des PME en autorisant une approche globale, que ce soit auprès d'entreprises à monoou à pluri-activités. D'autre part, ce mode de raisonnement réduit le risque d'inverser la question de recherche : la nature stratégique du projet étant en effet définie en fonction du niveau de risque couru par l'organisation, il doit s'agir d'une véritable rupture pour l'entreprise ${ }^{8}$. Le fait de donner ainsi au contexte d'investigation un caractère entrepreneurial favorise la perception de la fonction marketing comme clé de la mise en œuvre stratégique plutôt que comme reflet d'une "posture stratégique».

La première phase évoquée correspond au processus de décision. La perception et l'interprétation par le dirigeant de l'environnement constituent un contexte permanent qui influence le processus. Cette perception dépend de facteurs propres au dirigeant tels que ses valeurs personnelles, sa capacité de prévoir, son type de comportement (proactif ou réactif). Elle aboutit à la formulation d'un projet.

La phase suivante concerne la mise en œuvre du projet. En pratique, comme le montrent les deux cas réels présentés ci-après, le dirigeant peut être plus ou moins conscient de la nécessité d'assurer une cohérence entre les fonctions critiques d'ordre marketing et ses choix stratégiques? ${ }^{9}$.

8. Nous reviendrons ultérieurement sur les conditions d'admissibilité des projets observés.

9. Dans le cadre d'une première étude exploratoire, l'un des auteurs a mené une série d'entretiens semi-directifs auprès de dirigeants de PME en Aquitaine. Les principaux points abordés au cours de l'interview concernaient les origines, les modes de décisions et de mise en œuvre des choix récents considérés comme stratégiques par le dirigeant. 


\section{Le cas de l'entreprise D (une volonté d'intégration du marketing dans la stratégie).}

Cette entreprise spécialisée dans le négoce de fournitures industrielles appuie son développement sur une stratégie d'extension de sa zone de chalandise. Il y a quelque temps, s'est présentée la possibilité de racheter un concurrent situé à environ $200 \mathrm{~km}$ du siège social. Cela représentait pour le dirigeant une opération stratégique, permettant d'envisager un développement auprès d'une clientèle nouvelle et géographiquement accessible. Sur le plan marketing, la société, consciente du rôle critique joué par la qualité des produits proposés, a conservé la même politique de différenciation : un accord de partenariat avec les fournisseurs a permis de maintenir l'exclusivité des marques commercialisées sur l'ensemble de la nouvelle zone de chalandise.

\section{Le cas de la société $B$ (une définition limitée des activités critiques)}

L'entreprise, spécialisée dans les alliages d'aluminium, fournit une illustration d'un développement grâce à une stratégie d'innovation : la mise au point d'une technologie de coulage lui a permis de pénétrer un nouveau marché de fabrication de pièces en sous-traitance. Mais le dirigeant ne perçoit pas les incidences de ce choix stratégique sur son organisation commerciale. Au lieu d'accorder une attention particulière, par exemple, à une formation adaptée de la force de vente, à la mise en place d'une politique tarifaire plus ferme ou à l'élaboration d'une politique de communication valorisante (ces trois points représentent, dans ce cas, les principales fonctions critiques), il tient un discours qui demeure d'ordre très général : "c'est la technique qui définit les besoins, les contraintes de chaque client, [...] on est bien sûr obligé d'avoir un recul par rapport à la technique, nous avons un service commercial, un suivi, des objectifs, la prospection...»

\subsection{Les difficultés de la mise en œuvre de la recherche}

Notre réflexion quant au choix d'un champ d'observation pratique nous a conduits à organiser notre proposition autour du concept central de projet stratégique de type entrepreneurial. Si ses caractéristiques peuvent être relativement cernées d'un point de vue théorique, quelles sont, en pratique, les conditions d'admissibilité des projets qui devront être observés?

La nature stratégique du projet doit correspondre à une volonté entrepreneuriale de tirer profit d'une occasion d'affaires perçue dans l'environnement. En pratique, il peut s'agir de projets très différents : par exemple, la décision de cibler un segment de marché émergent, d'effectuer une modification technique qui va permettre au produit de surpasser ses concurrents, de se tourner vers un marché étranger. À l'image de la distinction établie par Mintzberg entre les 
stratégies intentionnelles et réalisées (Bamberger, 1982), le critère qui guidera notre sélection sera lié au type de stratégie associée au projet. Compte tenu des objectifs de notre recherche, nous retiendrons uniquement les projets «intentionnels», qui guideront les décisions de l'entrepreneur dans le futur par opposition à un projet réalisé dont les conséquences ne pourraient être constatées qu'a posteriori.

C'est à ce stade de la réflexion qu'apparaît la première difficulté : comment repérer les projets susceptibles d'être observés alors que les risques de confusion des dirigeants, à cet égard, ne sont pas négligeables ? Certains peuvent, par exemple, considérer comme stratégique une décision qui ne reflète pas une véritable rupture dans l'activité de l'entreprise; d'autres, au contraire, peuvent n'accorder que peu d'importance à un projet dont les caractéristiques correspondent particulièrement aux conditions d'admissibilité (cas de l'entreprise spécialisée dans les travaux d'ingénierie mécanique et qui a souhaité s'orienter vers un nouveau métier: la maintenance).

Par ailleurs, certaines entreprises peuvent aussi mettre en œuvre des projets qui correspondent simultanément à plusieurs types de choix stratégiques (innovation et développement international, par exemple) ou qui, au contraire, ne permettent pas d'identifier clairement une option particulière. Dans ce cas, quelles fonctions critiques observer en priorité ?

Se pose enfin le problème de la mesure de la performance. Chaque projet constitue en effet un cas unique, avec une chronologie, une situation dans l'ensemble des activités de l'entreprise et un rythme d'évolution bien particuliers. Par ailleurs, la notion même de performance est liée à la réalisation des objectifs associés à chaque projet considéré. Or, ces objectifs diffèrent en fonction de l'entrepreneur (Laufer, 1975; Hornaday, 1990). La diversité des situations impose une approche systémique de chacune d'elles; il n'est pas possible de "plaquer» sur chaque cas une batterie d'indicateurs de performance, aussi perfectionnés soient-ils. Il faut, par contre, s'appuyer sur le conducteur principal du projet, l'entrepreneur, et tenter ainsi d'évaluer l'écart qu'il perçoit entre les résultats et les objectifs qu'il s'était fixés. La mesure perceptuelle de la performance pourrait constituer une voie possible, sous réserve d'une méthodologie appropriée.

Toutefois, le fait que le dirigeant puisse apprécier la performance de son projet suppose qu'il ait suffisamment de recul sur son expérience et qu'il accepte de reconnaître certaines failles dans la mise en œuvre de son projet (par exemple, une force de vente qui n'aura pas été assez renforcée). Dès lors, comment parvenir à recueillir des données fiables et objectives?

Le premier type de biais concerne donc la présentation de l'objet de la recherche auprès de la population observée. Le chercheur devra être 
extrêmement attentif à ne pas tomber dans le piège d'un questionnement (par exemple, sur des erreurs de décision ou l'échec du projet) trop direct qui provoquerait une réaction de contraction défensive ${ }^{10}$. Pour surmonter cette difficulté, il faut faire raconter au dirigeant, au cours d'une interview semi-directive, le déroulement du projet en tentant de lui faire exprimer les précautions prises, les difficultés rencontrées, les succès ou les déceptions vécus.

Un deuxième risque de biais peut provenir d'une généralisation trop poussée de la nature des fonctions critiques dont le suivi va être considéré comme significatif de l'intégration marketing/stratégie. Par exemple, dans le cas d'une entreprise de sous-traitance qui se lancerait dans la fabrication d'un produit propre, l'adaptation de l'organisation commerciale peut être considérée comme l'une des fonctions critiques. Mais selon les situations, cette réorganisation commerciale peut revêtir des modalités très différentes (élaboration d'un catalogue, embauche de vendeurs). Cette diversité potentielle des cas, même pour un seul type de projet, rend plus délicate encore l'appréciation de la notion de «suivi des fonctions critiques».

Une recherche qui s'inscrit dans une logique déductive a généralement pour objectif de tester des hypothèses ou de rechercher des régularités dans un phénomène en appliquant des méthodes de traitement statistique des données empiriques récoltées. Il s'agit en général d'informations concernant un grand nombre d'individus ou d'organisations recueillies à l'aide d'un questionnaire. La mise en place de ces procédures de validation suppose la construction préalable d'un modèle théorique et la formulation d'hypothèses que l'on cherche à réfuter ou à confirmer. Ce type de démarche nous paraît cependant prématuré en l'état actuel des connaissances sur l'objet de la recherche. Le champ de l'investigation nous semble, en effet, plus proche d'un contexte de découverte que de justification. Par ailleurs, les problèmes posés par les critères d'admissibilité des projets observés, les risques de biais et la nécessité d'une mesure perceptuelle de la performance justifient le choix d'une logique inductive plus adapté à un objectif de type exploratoire, à savoir rendre intelligible les comportements d'intégration, suggérer des relations, générer des concepts nouveaux. La démarche permettrait d'inférer, à partir de l'observation de données, des hypothèses ou des éléments de théorie. Dans cette optique, le cadre d'analyse du processus de décision entrepreneurial que nous avons proposé ne doit surtout pas constituer un a priori théorique. Il peut seulement fournir une aide à l'interprétation des données en constituant une.structure pour la démarche de recherche (Eisenhardt, 1989).

10. On pourrait, par exemple, songer à une question telle que «Avez-vous modifié votre produit ? » Mais ce type d'interrogation est loin d'être neutre dans le cas où l'échec du projet s'explique justement par une mauvaise adaptation. 
Cette démarche inductive doit être de nature qualitative. Elle va permettre de lever les contraintes liées au risque de biais, puisqu'elle autorise trois caractéristiques importantes pour notre programme: la découverte d'éléments difficilement identifiables de manière directive, une étude sur la durée et la compréhension des perceptions émises par les acteurs.

Une stratégie d'analyse par «études de cas» permettrait de saisir et de décrire le plus objectivement possible - en partant des faits détaillés - les spécificités des situations étudiées, à savoir les comportements entrepreneuriaux d'intégration stratégie marketing. Ces cas devront être choisis de manière à être représentatifs de la population observée, non pas sur le plan statistique, mais du point de vue de la diversité et de la complexité de l'objet de la recherche. Devront donc être prises en compte les entreprises ayant adopté des projets stratégiques différents de manière à constituer un champ d'investigation aussi diversifié que possible. $L$ 'analyse et la triangulation des données ainsi recueillies (interviews, observations des comportements des acteurs, étude de documents commerciaux ou comptables) permettraient de relever des divergences ou des similitudes entre les situations concrètes de manière à faire émerger une configuration qui ferait ensuite l'objet d'une procédure de validation ${ }^{11}$.

Si l'influence de la formalisation de la stratégie sur la performance de la PME demeure un sujet très controversé, la convergence des activités opérationnelles avec les principales orientations stratégiques de l'entreprise est, par contre, reconnue comme un facteur de compétitivité (d'Amboise et Bakanibona, 1990). Les mécanismes selon lesquels se réalise la congruence entre le plan stratégique et les principales fonctions de l'entreprise demeurent cependant un domaine mal connu et l'intérêt d'une recherche en ce sens n'est pas à démontrer. Nos intentions à cet égard consistaient surtout à poser le problème de la conception d'un programme de recherche approprié et à en souligner les difficultés. À l'aide des apports de la littérature et des premiers éléments d'une étude exploratoire, nous venons ainsi de suggérer des orientations qui sont, selon nous, essentielles. De nombreux travaux seront sans doute nécessaires afin de comprendre parfaitement ces phénomènes et d'être en mesure de proposer aux dirigeants de PME des cadres de réflexion réalistes et adaptés à leur processus de décision.

11. La procédure de validation en usage est la confirmation croissante qui permet de conclure, après que plusieurs expériences similaires aient été menées dans des contextes différents ou sur des objets différents, que la loi est « vraie » (Bergadaa et Nyeck, 1992). 


\section{Bibliographie}

AABY, N. et A.F. MCGANN (1989), «Corporate strategy and the role of navigational marketing », European Journal of Marketing , vol. 23, n 10, p. 18-31.

Allaire, Y. et M. Firsirotu (1990), «La planification stratégique: un contrat psychologique et économique », Revue Française de Gestion, janvier-février, p. 22-39.

BAMBERgER, I. (1982), «Les valeurs des dirigeants de PME et leurs influences sur le comportement stratégique et la performance de leurs entreprises », Économie et Société. Sciences de Gestion, p. 1353-1383.

Belley, A., L. DusSault et J. LoRRAin (1991), «Le plan d'affaires pour la création d'une entreprise », Revue organisation, vol. 1, no 1, p. 23-41.

BERGADAA, M. et S. NYECK (1992), «Recherche en marketing: un état des controverses », Recherche et Applications en Marketing, vol. 7, $\mathrm{n}^{\circ} 3$.

BRACKER, J.S. et J.N. PEARSON (1986), «Planning and financial performance of small mature firms », Strategic Management Journal, vol. 7, p. 503-522.

Bracker, J.S., B.W. Keats et J.N. Pearson (1988), «Planning and financial performance among small firms in growth industry », Strategic Management Journal, vol. 9, p. 591-603.

CARRIÈRE, J.B. (1990), « La vision stratégique en contexte de PME : cadre théorique et étude empirique », Revue Internationale PME, vol. 3, nos 3-4, p. 301-327.

Chicha, J., P.A. Julien, M. MARChesnay (1990), «Processus et procédures stratégiques dans les PME », en allemand dans Internationales Gewerbearchiv, 3.

D'Amboise, G. et A. BAKANiBona (1990), «La planification dans les PME: une synthèse des résultats empiriques. Conclusions et recommandations ", Revue Internationale PME, vol. 3, $\mathrm{n}^{\circ} 2$, p. 147-167.

D’Amвoise, G. (1989), «Planification stratégique dans les PME: des modèles émergents de la littérature », Revue PMO, vol. 4, n 2 .

D' Amboise, G. et C. ForTIN (1989), «Proposition d'une grille de classification pour l'identification de la logique stratégique des petites et moyennes entreprises », Cahiers de recherche de la Faculté des sciences de l'administration de l'Université Laval, 32 p.

DeBrinay, G. (1990), «Les nouvelles lois de la compétitivité », Harvard L'expansion, printemps.

Dubois, P.L. et A. JOLIBERT (1992), Le marketing: fondements et pratique, Paris, Economica, $1^{\text {re }}$ édition 1989.

EISENHARDT, K. (1989), «Building theories from case study research », Academy of Management Review, vol. 14, $\mathrm{n}^{\circ} 4$. 
FILION, L.J. (1991), «Vision and relations : elements for an entrepreneurial model », International Small Business Journal, vol. 9, $\mathrm{n}^{\circ} 2$, p. 26-40.

HIRIgOYEN, G. (1993), "Nouvelles approches du lien stratégie-finance», Revue Française de Gestion, janvier-février, p. 64-73.

HORNADAY, R.W. (1990), «Dropping the E-words from small business research : an alternative typology », Journal of Small Business Management, vol. 28, $\mathrm{n}^{\circ} 4$, p. 22-34.

JOFFRE, P. et G. KOENIG (1992), Gestion stratégique. L'entreprise, ses partenairesadversaires et leur univers, Paris, Éditions Litec, 200 p.

Julien, P.A. et M. MARChESNAY (1988), La petite entreprise, Paris, Vuibert.

KoENIG, G. (1990), Management stratégique. Vision, manœuvres et tactiques, Paris, Nathan, 390 p.

LAMBIN, J.J. (1989), Le marketing stratégique. Fondements, méthodes et applications, Paris, McGraw-Hill, 450 p.

LAUFER, J. (1975), «Comment on devient entrepreneur », Revue Française de Gestion, novembre-décembre, p. 11-26.

LEO, P.Y., M.C. MONNOYER et J. PhILIPPE (1989), PME, stratégies internationales, Paris, Economica.

MCDANIEL, S.W. et J.W. KolaRI (1987), «Marketing strategy implications of the Miles and Snow strategic typology », Journal of Marketing, $\mathrm{n}^{\circ} 4$, p. 19-31.

MARTINET, A.C. (1988), «Les discours sur la stratégie d'entreprise », Revue Française de Gestion, janvier-février, p. 49-60.

MinTZBERG, H. (1990), «The design school: reconsidering the basic premises of strategic management », Strategic Management Journal, vol. 11, p. 171-195.

PORTER, M. (1991), « Towards a dynamic theory of strategy », Strategic Management Journal, vol. 12, p. 95-117.

RAYMOND, M.A. et H.C. BARKSDALE (1989), «Corporate strategic planning and corporate marketing : towards an interface », Business Horizons, septembreoctobre, p. 43-48.

REID, D. (1989), «Operationalizing strategic planning», Strategic Management Journal, vol. 10, p. 553-567.

Robinson, R.B., J.A. Pearce, G.S. Vozikis et T.S. Mescon (1984), «The relationship between stage of development and small firm planning and performance », Journal of Small Business Management, vol. 22, $\mathrm{n}^{\circ} 2$ 2, p. 45-52.

SAPORTA, B. (1986), Stratégies pour la PME, Paris, Montchrestien. 
Schrader, C.B., C.L. Mulford et V. BlackBurN (1989), «Strategic and operational planning, uncertainty and performance in small firms », Journal of Small Business Management, vol. 27, nº 4, p. 45-60.

STASCH, S.F. et J.L. WARD (1989), «Evaluating agressive marketing strategies for smaller share firms », Marketing Intelligence and Planning, ${ }^{\mathrm{os}} 7-8, \mathrm{p} .4-15$.

STRATÉGOR (1988), Stratégie, structure, décison, identité, Paris, Interéditions, 1988.

WALKER, O.C. et R.W. RUEKERT (1987), « Marketing's role in the implementation of business strategies : a critical review and conceptual framework», Journal of Marketing, juillet, p. 15-33. 